\title{
CONGENITAL MULTISEGMENTAL LYMPHATIC DYSPLASIA WITH SYSTEMIC INVOLVEMENT - EVALUATING PRIMARY LYMPHOEDEMA
}

\author{
Afthab Jameela Wahab ${ }^{1}$, V. Anandan'2, K. P. Saradha ${ }^{3}$, Nithya Baskar ${ }^{4}$ \\ ${ }_{1}^{1}$ Associate Professor, Department of Dermatology, Stanley Medical College. \\ ${ }^{2}$ Professor, Department of Dermatology, Stanley Medical College. \\ ${ }^{3}$ Associate Professor, Department of Dermatology, Stanley Medical College. \\ ${ }^{4} J u n i o r$ Resident, Stanley Medical College.
}

\section{ABSTRACT}

A ten-year old girl presented with unilateral swelling of right half of her body since birth. She developed itchy, oozing, painful skin lesions over right lower leg for the past three months. Clinical examination and investigations revealed unilateral lymphoedema with elephantiasis nostras verrucosa cutis and visceral involvement in the form of pericardial effusion, ascites and intestinal lymphangiectasia. She was diagnosed as a case of multisegmental lymphatic dysplasia with systemic involvement. We report this rare case of primary lymphoedema, highlighting the approach to a case of primary lymphedema.

\section{KEYWORDS}

Primary Lymphedema, Multisegmental Lymphatic Dysplasia, Lymphangiectasia.

HOW TO CITE THIS ARTICLE: Afthab Jameela Wahab, V. Anandan, K. P. Saradha, Nithya Baskar. "Congenital Multisegmental Lymphatic Dysplasia with Systemic Involvement - Evaluating Primary Lymphoedema." Journal of Evolution of Medical and Dental Sciences 2015; Vol. 4, Issue 100, December 14; Page: 16580-16583, DOI: 10.14260/jemds/2015/2469

\section{INTRODUCTION}

Primary lymphoedema results from an inherent developmental abnormality of the lymphatic system. Multisegmental Lymphatic Dysplasia with Systemic Involvement (MLDSI), a subtype of primary lymphedema, is characterized by a segmental pattern of lymphoedema affecting different body parts associated with systemic involvement.

\section{CASE REPORT}

A 10-year-old girl, who had hypertrophy of the entire right half of the body since birth presented with painful, itchy, oozing skin lesion over her right lower leg of three months duration (Figure-1).

She was the second child of non-consanguineous parents with a birth weight of $6.5 \mathrm{~kg}$, delivered by caesarean section. The hemihypertrophy had increased proportionately to her growth, uninfluenced by postural changes. She had developed recurrent cellulitis over her right leg swelling for which she was on intermittent treatment.

Patient gives history of recurrent upper respiratory tract infections. There was no history suggestive of cardiovascular, gastrointestinal and central nervous system involvement clinically.

The patient's vital signs, general and systemic examination was normal. There was increased girth of right half of face and body and right limbs indicating right unilateral lymphedema with soft tissue hypertrophy. There was no warmth or tenderness and no length discrepancy between right and left upper and lower limbs.

Financial or Other, Competing Interest: None.

Submission 17-11-2015, Peer Review 18-11-2015,

Acceptance 07-12-2015, Published 14-12-2015.

Corresponding Author:

Dr. Afthab Jameela Wahab,

R35, Flat 14, Nilgiri Apartments,

T S Krishna Nagar, Mugappair,

Chennai- 600037.

E-mail: afthabjameela@hotmail.com

DOI:10.14260/jemds/2015/2469
The swelling over the right lower limb was non-pitting, non-pulsatile, non-reducible and non-compressible. T Department of Dermatology here was no bruit, thrill or varicose veins. The dorsal aspect of lower third of right leg and foot showed elephantiasis nostras with hyperkeratotic, rugose, verrucous plaque of size $20 \times 15 \mathrm{~cm}$ with fissuring, crusting and erosion and milky white discharge (Figure-2). Kaposi-Stemmer's sign was positive. There were multiple discrete vesicles over right lumbar region, left lower back and right earlobe. Gait, IQ, sensory and motor examination was normal.

Routine hemogram, urine analysis, liver and renal function tests and lipid profile were normal. Ultrasonography revealed pericardial effusion and ascites. Oesophagogastroduodenoscopy showed inflamed duodenal bulb, nodules in the entire duodenum and granular white milky spots from bulb to second part of duodenum-D2.

Histopathology of biopsies taken from verrucous lesion showed pseudoepitheliomatous hyperplasia and dermal dilated lymphatic vessels (Figure-3), truncal vesicles showed microcystic lymphatic malformations and D2/D3 was consistent with intestinal lymphangiectasia. Doppler study of right limbs was normal with no evidence of vascular malformations. Ophthalmic examination revealed refractory error in the right eye. Chromosomal analysis showed XX genotype. Two attempts at MRI lymphangiography failed as the child did not cooperate.

The patient was diagnosed as Multisegmental Lymphatic Dysplasia with systemic involvement. The secondarily infected acute eczema was treated with appropriate antibiotics after culture and sensitivity. She was then referred to Surgical Gastroenterology for management of intestinal lymphangiectasia. 


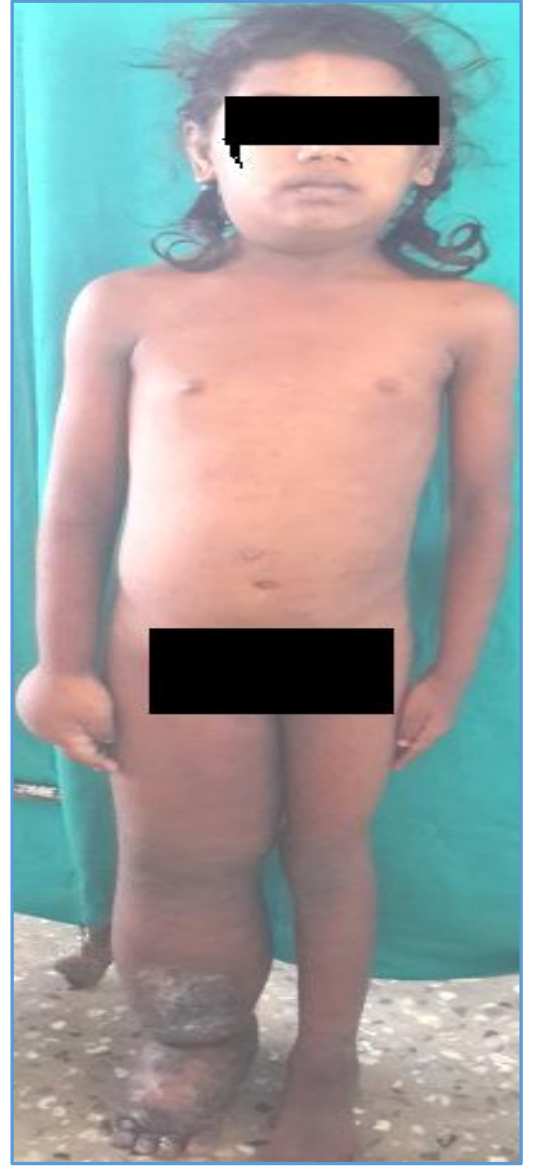

Fig. 1: Right-sided Unilateral Lymphoedema

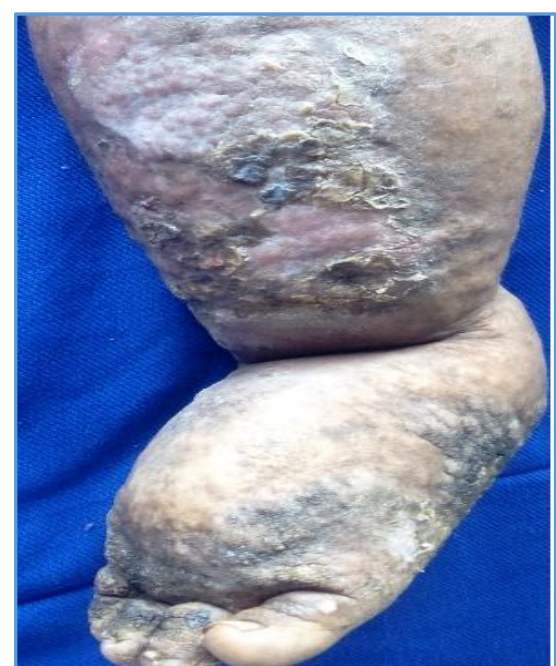

Fig. 2: Elephantiasis Nostras Verrucosa Cutis

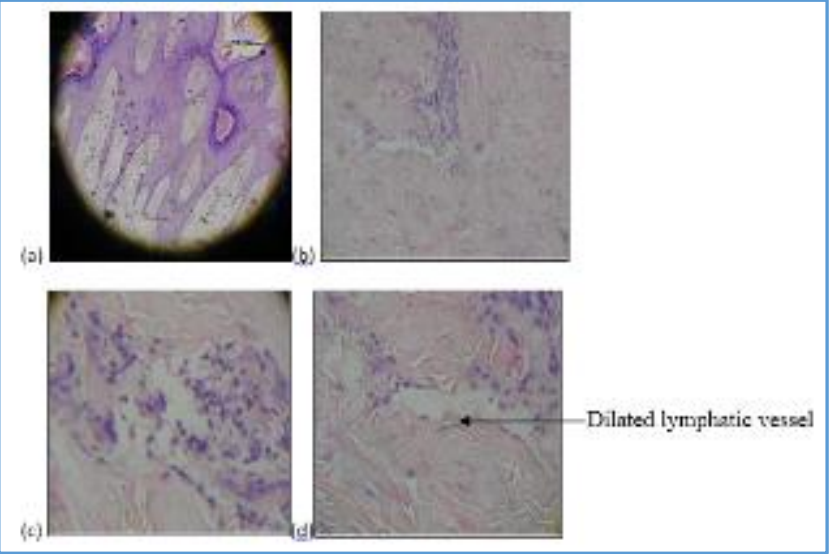

Fig. 3: Histopathology showing Pseudoepitheliomatous Hyperplasia (a) and Dilated Lymphatic Vessels (b, $c, d)$

\section{DISCUSSION}

Lymphoedema is defined as swelling of tissues resulting from accumulation of lymph caused by inadequate drainage. It may be primary or secondary. Primary lymphedema implies an intrinsic developmental or functional fault in lymph drainage. ${ }^{1}$ Secondary lymphedema occurs when previously normal lymphatics suffer from an external insult such as disease, infection, tumors, trauma or surgery with loss of functional capability. ${ }^{1}$

Primary lymphoedema may be classified into four types. ${ }^{2}$

1. Isolated primary lymphedema.

2. Primary lymphedema associated with systemic or visceral involvement.

3. Primary lymphedema associated with disturbed growth/ cutaneous/vascular anomalies.

4. Primary lymphedema as part of syndromes.

Isolated primary lymphoedema is lymphoedema confined to skin and soft tissue only. It may be of congenital onset $(<1$ year) or of late onset ( $>1$ year), further subtyping depending on the limb or segments involved.

\begin{tabular}{|c|c|c|c|c|c|}
\hline One Limb & $\begin{array}{l}\text { Lower limb } \\
\text { Non-familial } \\
\text { Unilateral }\end{array}$ & $\begin{array}{c}\text { Lower limb } \\
\text { Non- } \\
\text { familial } \\
\text { Bilateral }\end{array}$ & $\begin{array}{l}\text { Lower limb } \\
\text { Familial }\end{array}$ & $\begin{array}{c}\text { Lower limb } \\
\text { and } \\
\text { Genitalia }\end{array}$ & $\begin{array}{l}\text { Multiple } \\
\text { Segments }\end{array}$ \\
\hline $\begin{array}{c}\text { Congenital unisegmental } \\
\text { lymphedema }\end{array}$ & $\begin{array}{l}\text { Congenital } \\
\text { unisegmental } \\
\text { lymphedema }\end{array}$ & $\begin{array}{c}\text { Milroy } \\
\text { disease, } \\
\text { VEGFR3 } \\
\text { Milroy like } \\
\text { disease, } \\
\text { KIF11,VEGFC }\end{array}$ & $\begin{array}{c}\text { Milroy } \\
\text { disease, } \\
\text { VEGFR3 } \\
\text { Milroy like } \\
\text { disease, } \\
\text { KIF11,VEGFC }\end{array}$ & $\begin{array}{l}\text { Lower limb } \\
\text { + genital } \\
\text { lymphedema }\end{array}$ & $\begin{array}{c}\text { Congenital } \\
\text { multisegmental } \\
\text { lymphedema } \\
\text { without systemic } \\
\text { involvement }\end{array}$ \\
\hline
\end{tabular}


Milroy's disease, an autosomal dominant disease caused by failure of lymphangiogenesis secondary to inactivation of VEGFR-3 with familial, bilateral below-knee lymphoedema. ${ }^{3}$

\begin{tabular}{|c|c|c|c|c|c|c|}
\hline $\begin{array}{c}\text { Lymphedema } \\
\text { with } \\
\text { Distichiasis }\end{array}$ & $\begin{array}{c}\text { Lower } \\
\text { limbs } \\
\text { Familial }\end{array}$ & $\begin{array}{c}\text { Lower limbs } \\
\text { Non-familial } \\
\text { Bilateral }\end{array}$ & $\begin{array}{c}\text { Lower limbs } \\
\text { Non-familial } \\
\text { Unilateral }\end{array}$ & $\begin{array}{c}\text { Lower limb } \\
\text { and genitalia }\end{array}$ & 4-limb & Segmental \\
\hline $\begin{array}{c}\text { Lymphoedema- } \\
\text { Distichiasis } \\
\begin{array}{c}\text { Syndrome } \\
\text { FOXC2 }\end{array}\end{array}$ & $\begin{array}{c}\text { Meige } \\
\text { GJC2 }\end{array}$ & Meige like & $\begin{array}{c}\text { Late onset } \\
\text { unilateral leg } \\
\text { lymphoedema }\end{array}$ & $\begin{array}{c}\text { Lower limb }+ \\
\text { genital } \\
\text { lymphedema } \\
\text { GATA2 }\end{array}$ & $\begin{array}{c}\text { 4-Limb } \\
\text { lymphedem } \\
\text { a GJC2 }\end{array}$ & $\begin{array}{c}\text { Late onset uni } \\
\text { or multi- } \\
\text { segmental } \\
\text { lymphoedema }\end{array}$ \\
\hline \multicolumn{70}{|c|}{ Table 2: Late onset Lymphedema } \\
\hline
\end{tabular}

Lymphoedema-distichiasis syndrome is an autosomal dominant disease caused by mutations in FOXC2 (MFH-1). Congenital accessory eyelashes are present along the posterior eyelid borders. Lymphoedema may occur from puberty to fifth decade. 4 Meige's disease is familial, mild bilateral below-knee lymphoedema developing at or soon after puberty in adolescent female. ${ }^{5}$

\section{Primary lymphedema associated with systemic or visceral involvement}

Individuals have widespread developmental abnormality of the lymphatic system. There may be prenatal hydrothorax, hydrops fetalis, dysmorphic facial features with epicanthic folds, broad nasal bridge, neck webbing and low set ears. Postnatal pericardial and pleural effusions, chylous ascites and pulmonary and intestinal lymphangiectasia may develop. The lymphedema may be multisegmental or generalized. Accordingly, they can be further divided into two categories.

Multisegmental Lymphatic Dysplasia with Systemic Involvement (MLDSI)

Associated with the above systemic involvement, there is congenital segmental lymphoedema affecting different body parts, probably due to somatic mosaicism. There may be ipsilateral hemifacial and conjunctival oedema. Intelligence is normal. There are no associated structural abnormalities. Sibling and offspring recurrence risk is low. ${ }^{2}$
Generalized Lymphatic Dysplasia (GLD)- Hennekam or lymphangiectasia- lymphoedema-mental retardation syndrome.

An autosomal recessive disease due to mutations in collagen and calcium binding EGF-domain 1 (CCBE1). ${ }^{6}$ it is characterized by uniform, widespread lymphoedema affecting all segments of the body from birth to 12 years, intestinal lymphangiectasia, hypoproteinemia, learning difficulties and characteristic oriental facies.

Primary Lymphedema associated with disturbed growth and/or cutaneous/vascular anomalies

Lymphoedema and lymphatic malformations can be seen in conjunction with growth disturbances or vascular/cutaneous anomalies resulting from somatic mosaicism.

\begin{tabular}{|c|c|}
\hline Syndromes & Clinical Features \\
\hline $\begin{array}{c}\text { CLOVES syndrome } \\
\text { PIK3CA }\end{array}$ & $\begin{array}{c}\text { Congenital lipomatous overgrowth, vascular malformations, epidermal } \\
\text { naevi, skeletal abnormalities }\end{array}$ \\
\hline Klippel-Trenaunay syndrome E133K & $\begin{array}{l}\text { Lymphoedema, capillary malformation, venous disease, limb } \\
\text { overgrowth }\end{array}$ \\
\hline Parkes Weber syndrome RASA1 & $\begin{array}{l}\text { High flow capillary-Arteriovenous Malformation (AVM) or capillary- } \\
\text { lymphatic-AVM, vascular stain, limb overgrowth }\end{array}$ \\
\hline $\begin{array}{l}\text { Proteus syndrome } \\
\text { AKT1, P10 }\end{array}$ & $\begin{array}{c}\text { Asymmetrical overgrowth of any body part, macrodactyly, } \\
\text { palmoplantar cerebriform overgrowth, verrucous epidermal naevi, } \\
\text { lymphangiomatous swelling }\end{array}$ \\
\hline $\begin{array}{l}\text { WILD syndrome } \\
\text { Sporadic }\end{array}$ & Warts, immunodeficiency, lymphoedema, anogenital dysplasia \\
\hline Maffucci's syndrome. ${ }^{7}$ Sporadic & $\begin{array}{l}\text { Haemangioma, venous malformations, cavernous lymphangiomas, } \\
\text { dyschondroplasia, enchondromas }\end{array}$ \\
\hline
\end{tabular}

\section{Primary lymphedema as a part of syndromes}

Turner's syndrome 45XO Chromosomal testing is essential in neonates/children with primary lymphoedema.

Noonan's syndrome PTPN11.

Hypotrichosis-lymphoedema -telangiectasia syndrome

SOX18.

Microcephaly-lymphoedema-chorioretinal dysplasia KIF11. ${ }^{8}$

Cholestasis- lymphoedema syndrome (Aagenaes's syndrome). Yellow nail syndrome.

\section{CONCLUSION}

An algorithmic approach to evaluate a case of primary lymphoedema would be to first assess the extent of lymphedema, systemic/visceral lymphatic involvement and then to exclude associated syndromes, growth disturbances, vascular/cutaneous anomalies. In our case associated syndromes were ruled out by clinical examination and chromosomal analysis. Further investigations excluded vascular malformations, cutaneous anomalies and growth disturbances. Systemic lymphatic involvement (intestinal lymphangiectasia, pericardial effusion, ascites) ruled out isolated primary lymphoedema. Unilateral congenital lymphoedema with normal intelligence excluded GLD, confirming the diagnosis of "Multisegmental lymphatic dysplasia with systemic involvement." This rare-case report attempts at highlighting an algorithmic approach in evaluating primary lymphoedema.

\section{REFERENCES}

1. Mortimer PS (1998). The pathophysiology of lymphedema. Cancer, 83:2798-2802.

2. Connell FC, et al. The classification and diagnostic algorithm for primary lymphatic dysplasia: an update from 2010 to include molecular findings. Clin Genet 2013;84:303-314. 
3. Rrthum A, Karkkainen MJ, Devriendt K, Alitalo K, Vikkula M. Congenital Hereditary Lymphedema Caused by a Mutation That Inactivates VEGFR3 Tyrosine Kinase. American Journal of Human Genetics. 2000;67(2):295-301.

4. Mangion et al., 1999. A gene for lymphoedemadistichiasis maps to 16q24.3. am j hum genet, vol 65, 1999, pp427-432.

5. Meige H. Dystrophie oedemateuse héréditaire. Presse Méd 1898;6:341-3.

6. Alders $M$, et al. Mutations in CCBE1 cause generalized lymph vessel dysplasia in humans. Nat Genet 2009;41:1272-1274.
7. Carlton A, St Elkington JC, Greenfi eld JG, et al. Maffucci's syndrome. QJM 1942;11:203-8.

8. Ostergaard P, Simpson MA, Mendola A, et al. Mutations in KIF11 Cause Autosomal-Dominant Microcephaly Variably Associated with Congenital Lymphedema and Chorioretinopathy. American Journal of Human Genetics.2012;90(2):356-362. doi:10.1016/j.ajhg.2011.12.018. 